\title{
Modulation of hypothalamic S6K1 and S6K2 alters feeding behavior and systemic glucose metabolism
}

\author{
Mariana Rosolen Tavares 1,2, Simone Ferreira Lemes², Thais de Fante², Cristina Saenz de Miera4, \\ Isadora Carolina Betim Pavan1,2, Rosangela Maria Neves Bezerra1, Patricia Oliveira Prada3, \\ Marcio Alberto Torsoni², Carol Fuzeti Elias ${ }^{4}$ and Fernando Moreira Simabuco ${ }^{1,2}$ \\ ${ }^{1}$ Multidisciplinary Laboratory of Food and Health (LABMAS), School of Applied Sciences (FCA), University of Campinas (UNICAMP), Limeira, \\ São Paulo, Brazil \\ 2Laboratory of Metabolic Disorders (LABDIME), School of Applied Sciences (FCA), University of Campinas (UNICAMP), Limeira, São Paulo, Brazil \\ ${ }^{3}$ Laboratory of Molecular Research in Obesity (LABIMO), School of Applied Sciences (FCA), University of Campinas (UNICAMP), Limeira, São Paulo, Brazil \\ ${ }^{4}$ Department of Molecular and Integrative Physiology, University of Michigan, Ann Arbor, Michigan, USA
}

Correspondence should be addressed to F M Simabuco: simabuco@gmail.com

\begin{abstract}
The mTOR/S6Ks signaling is one of the intracellular pathways important for metabolic control, acting both peripherally and centrally. In the hypothalamus, mTOR/S6Ks axis mediates the action of leptin and insulin and can modulate the expression of neuropeptides. We analyzed the role of different S6Ks isoforms in the hypothalamic regulation of metabolism. We observed decreased food intake and decreased expression of agouti-related peptide (AgRP) following intracerebroventricular (icv) injections of adenoviral-mediated overexpression of three different S6Ks isoforms. Moreover, mice overexpressing p70-56K1 in undefined periventricular hypothalamic neurons presented changes in glucose metabolism, as an increase in gluconeogenesis. To further evaluate the hypothalamic role of a less-studied S6K isoform, p54-S6K2, we used a Cre-LoxP approach to specifically overexpress it in AgRP neurons. Our findings demonstrate the potential participation of S6K2 in AgRP neurons regulating feeding behavior.
\end{abstract}

Key Words
- metabolism
- obesity
mTOR
- S6Ks
- hypothalamus
- neuroendocrinology

\section{Background}

Obesity is an important worldwide public health issue, consisting in a concerning matter, due to its association with several chronic diseases, including type 2 diabetes, cardiovascular diseases, stroke, dyslipidemias, osteoarthritis, and some types of cancer (James et al. 2006, Tremblay \& Lachance 2017). Thus, there is a great demand for research involving metabolic processes related to the obesity onset. Hypothalamus is an important region of the central nervous system (CNS) for controlling the metabolism of the entire body, being responsible for integrating hormonal and nutritional signals from the periphery and for coordinating appropriate responses associated with the individual nutritional status (Bouret \& Simerly 2004, Myers \& Olson 2012, Schneeberger et al. 2014, Krashes et al. 2016, Dodd \& Tiganis 2017, Timper \& Brüning 2017).

Authors of several studies suggested that the mTOR/ S6K signaling pathway, in the arcuate nucleus of the hypothalamus (ARH), mediates part of metabolic effects of insulin and leptin with consequent reduction in food intake and increase in energy expenditure (Cota et al. 2006, 2008, Kahn \& Myers 2006, Blouet et al. 2008, 
Muta et al. 2015). These effects were associated with decreased expression of orexigenic neuropeptides, agoutirelated peptide (AgRP) and neuropeptide Y (NPY), in ARH neurons (Blouet et al. 2008). Even though other researchers did not verify changes in food intake or body weight in rodents with altered hypothalamic S6K1, they documented the importance of altering hypothalamic S6K1 expression for the metabolic functioning of peripheral tissues (Ono et al. 2008, Smith et al. 2015).

S6K1 overexpression in rats' mediobasal hypothalamus (MBH) decreased the ability of hypothalamic insulin to suppress hepatic glucose production (Ono et al. 2008). Conversely, expression of a dominant negative S6K1 restored the suppression of hepatic gluconeogenesis in response to hypothalamic insulin (Ono et al. 2008). Specific deletion of S6K1 in pro-opiomelanocortin (POMC) neurons also impaired the hepatic glucose production (Smith et al. 2015). Thus, it seems that the hypothalamic mTOR/S6K signaling pathway has both direct and indirect effects on energy metabolism, which requires further investigation.

Most of the data available on literature regarding the $\mathrm{mTOR} / \mathrm{S} 6 \mathrm{~K}$ role in the central control of metabolism have focused on S6K1, specifically on the p70-S6K1 isoform. Because $\mathrm{S} 6 \mathrm{~K} 1$ and $\mathrm{S} 6 \mathrm{~K} 2$ have a high degree of structural homology and the assumption that both proteins have identical biological functions, research focused on S6K2 has been highly neglected (Pardo \& Seckl 2013). However, some authors have indicated there are functional differences between S6Ks isoforms (Tavares et al. 2015).

The 40S ribosomal protein S6 is considered the main S6K1 and S6K2 substrate. Both kinases are necessary to the complete phosphorylation of S6, but S6K2 has a more important participation in this role (Pende et al. 2004). We previously verified that S6K1 and S6K2 present distinct interactomes (Pavan et al. 2016) and others have shown that S6K2 protein expression varies considerably between different tissues, like kidney and adrenal gland, while S6K1 protein expression is more uniform (Nardella et al. 2011).

Analysis of the literature reveals a lack of studies focusing on the S6K2 expression in the hypothalamus and other metabolic tissues as well as its role in the metabolism control (Tavares et al. 2015). An important study has shown that S6K1-knockout mice presented lower body mass, whereas S6K2-knockout mice presented higher body mass compared to wild-type (WT) mice during postnatal growth (Pende et al. 2004). These differences between S6Ks isoforms highlight the importance of researches focusing on different isoforms, especially regarding the less-studied S6K2 isoform.

In this study, we found that both hypothalamic S6K1 isoforms (p70-S6K1 and p85-S6K1) and the S6K2 isoform (p54-S6K2) have effects on food intake, and the p70-S6K1 isoform has an additional impact on the regulation of hepatic glucose homeostasis. We further selectively evaluated the role of S6K2 isoform in AgRP neurons, since these neurons are well-known targets of leptin and insulin role in energy balance and glucose homeostasis (Xu et al. 2018).

\section{Materials and methods}

\section{Animals}

Swiss male mice (7-9 weeks old) were provided by the Multidisciplinary Center for Biological Research, University of Campinas (CEMIB). Mice were kept in a room with controlled temperature $\left(22-24^{\circ} \mathrm{C}\right)$ and a 12-h light/darkness cycle, with ad libitum access to water and food. Mice were fed with standard chow (NuvilabR CR-1, Nuvital). All experiments involving animal procedures followed the NIH 'Guide for the Care and Use of Laboratory Animals', and the guidelines of the Brazilian Institute for Animal Experimentation. Experiments were approved in advance by the Research Ethics Committee of the University of Campinas (Protocol no.: 3579-1 (A)).

Male AgRP-Cre (JAX mice, stock number 012899) and female wild-type (WT; JAX mice, stock number 000664) mice were kept in the animal facility of the University of Michigan under a standard light/darkness cycle and temperature $\left(21-23^{\circ} \mathrm{C}\right)$, with ad libitum access to water and standard chow (2016 Teklad Global 16\% Protein Rodent Diet, Envigo). The AgRP-Cre mouse colony was generated by crossing AgRP-Cre males with WT female mice. All mice were tail genotyped before experiments using primers described in Table 1 . Tail DNA was extracted using Sigma REDExtract-N-Amp tissue PCR kit, and genotype was defined by the presence of a 213-bp band using a 100-bp DNA ladder (New England Biolabs). All procedures and experiments were carried out according to the guidelines established by the NIH 'Guide for the Care and Use of Laboratory Animals', and approved by the Institutional Animal Care \& Use Committee of the University of Michigan (IACUC; protocol no.: 00008712). All efforts were made to minimize the number of used animals. 
Table 1 List of primers.

Primer/gene
Wild-type
Generic Cre
Ywhaz
Agrp
Pomc
Npy
G6pc

\begin{tabular}{l}
\hline Forward $\left(5^{\prime}-3^{\prime}\right)$ \\
\hline Genotyping \\
ACCTGTTCCACGCACAGTCACA \\
TGCTTCTGTCCGTTTGCCGGT \\
RTqPCR \\
GAACTCCCCAGAGAAAGCCT \\
CGGAGGTGCTAGATCCACAGA \\
AGAACGCCATCATCAAGAAC \\
CACCAGACAGAGATATGGCAAG \\
GCGCAGCAGGTGTATACTATG \\
\hline
\end{tabular}

Reverse $\left(5^{\prime}-3^{\prime}\right)$
AGCTGGCCAAATCTCAGAGCTGC
GTGAAACAGCATTGCTGTCAC
CCGATGTCCACAATGTCAAGT
AGGACTCGTGCAGCCTTACAC
AAGAGGCTAGAGGTCATCAG
TCACCACATGGAAGGGTCTT
CGTTCAAACACCGGAATCCA

\section{Cloning and preparation of viral vectors}

The gene of p70-S6K1, p85-S6K1 and p54-S6K2 proteins (RPS6KB1 and RPS6KB2) were cloned in pShires plasmid and subcloned in pAdeno-X plasmid. Adenoviral vectors for S6K1 and S6K2 isoforms were generated using Adeno- $X^{\mathrm{TM}}$ Expression System 1 (Clontech). Adenoviruses were produced in HEK293 cells and purified in a double gradient of cesium chloride (Armelini et al. 2005). Adenoviral titers were measured by endpoint dilution assay with 1:3 serial dilutions on a 96-well plate of HEK293 cells.

For Cre-dependent adeno-associated virus serotype 8 (AAV8) vectors, the gene of the p54-S6K2 protein fused with the FLAG peptide DNA sequence was cloned in a pAAV plasmid (Pavan et al. 2016). AAV8-control and AAV8-S6K2 viruses were packaged at the Vector Core facility of the University of Michigan.

\section{In vitro functional validation of viral vectors}

Hypothalamic GT1-7 cells were transduced with Ad-p85-S6K1 using a multiplicity of infection (MOI) of 10,000. Cells were visualized and images were captured using Leica DMI 4000B immunofluorescence microscope (Leica Microsystems).

HEK293 cells were infected with Ad-p70-S6K1, Ad-p85-S6K1, Ad-p54-S6K2, and Ad-EGFP (MOI=10). After $48 \mathrm{~h}$, cells were serum-starved for $2 \mathrm{~h}$, treated or not with $100 \mathrm{nM}$ rapamycin for $15 \mathrm{~min}$, and stimulated with $10 \mu \mathrm{g} / \mathrm{mL}$ of insulin for 15 more minutes. Cells were harvested and analyzed by Western blot as described next. For adeno-associated viral constructs, HEK293 cells were co-transfected with Cre-expressing plasmid (pCAG) or nonCre-expressing plasmid (pShires), and pAAV-S6K2 or pAAVEGFP, and then harvested and analyzed by Western blot, as described next.

\section{Stereotaxic surgery}

For intracerebroventricular (icv) injections, stereotaxic surgery was performed under ketamine/xylazine anesthesia and, after the loss of reflexes, mice were positioned in stereotaxic apparatus (Stoelting). Adenoviral vectors overexpressing the S6Ks isoforms (Ad-p70-S6K1: $4.22 \times 10^{9}$ PFU; Ad-p85-S6K1: $1.33 \times 10^{13}$ PFU; S6K2: $\left.7.5 \times 10^{13} \mathrm{PFU}\right)$ and EGFP $\left(3.16 \times 10^{12} \mathrm{PFU}\right)$ were injected $(2 \mu \mathrm{L})$ into the third ventricle with a 30-gauge needle connected to a Hamilton syringe $(5 \mu \mathrm{L})$ under adapted coordinates $(-0.5 \mathrm{~mm}$ anteroposterior; $0.2 \mathrm{~mm}$ lateral; $-5.0 \mathrm{~mm}$ depth, Ignacio-Souza et al. 2014).

For bilateral injections into the ARH, AgRP-Cre mice were anesthetized with $2 \%$ isoflurane and after the loss of reflexes, they were placed in a stereotaxic apparatus. The AAV-S6K2 or control vector was bilaterally injected (100nL each side) under pre-established coordinates $(-1.4 \mathrm{~mm}$ anteroposterior; $\pm 0.3 \mathrm{~mm}$ lateral; $-5.5 \mathrm{~mm}$ depth, $\mathrm{Xu}$ et al. 2018).

\section{Metabolic studies}

Metabolic and physiologic parameters were measured for 1 day and 5 days after adenovirus injections using indirect calorimetry system Oxymax/CLAMS (Columbus), at the University of Campinas.

For hypothalamic S6K2-specific modulation, experiments were performed in two different cohorts of males and females mice each. Metabolic and physiologic parameters were analyzed for a week, 6 weeks after AAV injections in a first cohort, and 13 weeks after AAV injections in a second cohort. After 3 days of measurements, mice were fasted overnight $(16 \mathrm{~h})$ and refed in the morning. Data were collected by the Animal Phenotyping Core facility of the University of Michigan. Since injections were variable and the number of mice obtained in each cohort yielded a small $n$, data were analyzed combining both cohorts.

\section{Intraperitoneal glucose and pyruvate tolerance test (ipGTT and ipPTT)}

For ipGTT, mice were fasted overnight $(12 \mathrm{~h})$, refed for $2 \mathrm{~h}$, followed by 4 -h fasting and an intraperitoneal 
injection of glucose $(1 \mathrm{~g} / \mathrm{kg})$. For ipPTT, mice were fasted overnight $(12 \mathrm{~h})$, followed by intraperitoneal injection of sodium pyruvate $(2 \mathrm{~g} / \mathrm{kg})$. Blood samples were collected by tail vein before and after the injection at established time intervals $(0,15,30,60$ and $120 \mathrm{~min})$. The concentration of plasma glucose was determined using Accu Check equipment (Bayer).

\section{Immunofluorescence}

Mice were perfused with $4 \%$ paraformaldehyde solution (PFA). Brains were extracted and fixed in 4\% PFA, and subsequently embedded in Tissue-Tek (Sakura), frozen and cut into $30 \mu \mathrm{m}$ thick coronal sections. Sections were incubated in blocking solution (1\% bovine albumin; Sigma-Aldrich) for $60 \mathrm{~min}$, followed by incubation with specific primary antibodies overnight at $4^{\circ} \mathrm{C}$. The used primary antibodies were:Anti-S6K1 1:500 (\#2708, Cell Signaling Technology), Anti-S6p 1:200 (\#2215, Cell Signaling Technology), and anti-FLAG 1:1000 (\#8146, Cell Signaling Technology). Sections were washed and incubated with appropriate secondary antibodies for 90 min. For immunofluorescence, the secondary Alexa 568-conjugated donkey anti-rabbit 1:500 (A10042, Life Technologies) and DAPI for nuclear labeling (1:1000) were used. For immunoperoxidase (anti-FLAG), sections were incubated in biotin-conjugated donkey anti-rabbit 1:1000 (Jackson Laboratories) for $1 \mathrm{~h}$ and avidin-biotin complex 1:500 (Vector Labs) for $1 \mathrm{~h}$. Diaminobenzidine (DAB, Sigma) was used as a chromogen. Sections were placed on slides previously treated with poly-L-lysine or gelatin for tissue adherence and coverslipped with Entellan (Merk) or DPX (immunoperoxidase, following dehydration). Hypothalamic sections were visualized and images were captured using Leica DMI 4000 B (Leica Microsystems) or AxioImager M2 (Zeiss) microscope.

\section{Real-time PCR}

Total RNA was isolated from frozen hypothalamus wedges (3-5 mg) and liver tissue (100 mg) using TRIzol (Invitrogen) according to the manufacturer's instructions. Extracted RNA was quantified using a NanoDrop 2000 (NanoDrop). RNA was used as a template for first-strand cDNA synthesis using High-Capacity cDNA Reverse Transcription Kit (Thermo Fisher Scientific). Quantitative PCR was performed using SYBR Green PCR Master Mix (Applied Biosystems) in a 7500 Fast Real-Time PCR System (Applied Biosystems). Each reaction was performed in a final volume of $12.5 \mu \mathrm{L}$ containing $1.0 \mu \mathrm{L}$ of cDNA (sample), $1.25 \mu \mathrm{L}$ of primer forward, $1.25 \mu \mathrm{L}$ of primer reverse, and $6.25 \mu \mathrm{L}$ of SYBR Green PCR Master Mix (Applied Biosystems). Forward and reverse primer pairs are listed in Table 1. Samples without cDNA were included as negative controls. Ywhaz was used as a housekeeping gene. All samples (unknown and standard curves) were assessed in triplicate. Quantification of transcript for each gene was obtained by the delta-delta cycle threshold method $(\Delta \Delta \mathrm{Ct})$. Fold change of mRNA in the experimental group concerning the control group was determined by $2^{-\Delta \Delta C t}$. Data are shown as a percentage of the relative mRNA expression of the control group.

\section{Western blot analysis}

Hypothalamus of mice injected with adenoviruses $(n=3-4)$ and protein samples of HEK293 cells were fractionated by polyacrylamide gel electrophoresis containing SDS (SDS-PAGE) using a run buffer $(50 \mathrm{mM}$ Tris; $0.38 \mathrm{M}$ Glycine; $1.8 \mathrm{mM}$ EDTA; $0.1 \%$ SDS). Then, proteins were transferred to nitrocellulose membrane under a $20 \mathrm{~V}$ potential difference for $1 \mathrm{~h}$ using transfer buffer $(24.8 \mathrm{mM}$ Tris; $192 \mathrm{mM}$ Glycine; $10 \%$ Methanol). Membranes were blocked at room temperature for $2 \mathrm{~h}$ in $5 \%$ skim milk diluted in TBS-T buffer (10 mM Tris-HCl; $150 \mathrm{mM} \mathrm{NaCl}$; $0.02 \%$ Tween-20). Membranes were incubated at room temperature for $1 \mathrm{~h}$ or overnight with adequate dilutions of primary antibodies diluted in 5\% milk and in TBS-T. Membranes were washed three times for 10 min with TBS-T and incubated at room temperature for $1 \mathrm{~h}$ with secondary antibodies, diluted in 5\% skim milk and in TBS-T. After three 10-min washes with TBS-T, the membranes were treated with chemiluminescent reagents (Pierce ${ }^{\mathrm{TM}}$ ECL Western Blotting Substrate, Thermo Scientific). Membranes were revealed by automatic imaging systems or exposed to films. The intensities of the bands were quantified by densitometry using ImageJ software and normalized to $\alpha$-tubulin levels. Antibodies used for blotting were p70 S6 Kinase (1:1000 \#2708 Cell Signaling Technology), p70 S6 Kinase 2 (1:1000 \#14130 Cell Signaling Technology), S6 Ribosomal Protein (1:1000, \#2317 Cell Signaling Technology), Phospo-S6 Ribosomal Protein Ser240/244 (1:1000, \#2215 Cell Signaling Technology), FLAG (1:2000, \#F3165, Sigma), and Anti- $\alpha$-tubulin as a loading control (1:2000 \#CP06 Calbiochem).

\section{Statistical analysis}

Results are presented as mean \pm S.E.M. Single comparisons were analyzed using a two-tail Student's $t$-test following 
confirmation of normal distribution by the KolmogorovSmirnov test or by a nonparametric Mann-Whitney test. Statistical significance for all analyses was set at $P<0.05$. All statistical comparisons were performed using GraphPad Prism 8. Data were analyzed to determine outliers according to Grubb's test with statistical significance set at $P<0.05$ (https://www.graphpad.com/ quickcalcs/Grubbs1.cfm).

\section{Results}

\section{Overexpression of S6Ks isoforms in $\mathrm{MBH}$}

Adenoviral vectors coexpressing the EGFP reporter gene were used to overexpress the S6Ks isoforms in $\mathrm{MBH}$ through icv injections (Fig. 1A). We first tested the transduction of adenoviral vectors in GT1-7 hypothalamic cells. The successful Ad-p85-S6K1 transduction was observed by the presence of EGFP or green fluorescence (Fig. 1B). For functional analysis, we tested the Ad-p70-S6K1, Ad-p85-S6K1, Ad-p54-S6K2, and Ad-EGFP in HEK293 cells treated with rapamycin or insulin, an inhibitor and an activator of the mTORC1/S6K pathway, respectively. We observed that insulin increased S6 phosphorylation at higher levels in S6Ks overexpressing cells compared with EGFPcontrol cells (Fig. 1C). Rapamycin effect in S6Ks overexpressing cells was diminished compared with control cells (Fig. 1C).

After testing the adenoviral vectors in cells, we assessed their in vivo role by injecting each adenovirus construct icv (Fig. 2A). We observed that the expression of all adenoviral vectors was restricted to the third ventricle (3V) wall and adjacencies, that is, tanycytes, periventricular and ARH neurons, with a peak of expression after 5 days, but persisting for up to 10 days (Fig. 2B). We also verified increased S6 phosphorylation in periventricular and ARH regions resulting from adenoviral vectors injection (Fig. 2C). Another group of mice with icv injections were subjected to protein quantification using Western blot analysis. We found an increase in hypothalamic S6 phosphorylation 7 days after icv Ad-p70-S6K1, Ad-p85-S6K1 and Ad-p54-S6K2 injections compared with control Ad-EGFP icv-injected mice (Fig. 2D).

\section{Hypothalamic S6Ks isoforms regulate food intake}

We first investigated the role of hypothalamic S6Ks isoforms controlling physiological parameters. Body weight was measured for 10 days before and 7 days after injections of adenoviral vectors. No changes were observed between EGFP-control and S6Ks-injected mice (Fig. 3A). However, p85-S6K1- and p54-S6K2-injected mice did show a cumulative 24 -h decreased food intake (Fig. 3B). The analysis of the circadian pattern of food consumption showed that mice overexpressing p70-S6K1 and p85-S6K1 decreased food intake in the dark cycle (Fig. 3C), corroborating previous studies (Cota et al. 2006, 2008, Blouet et al. 2008, Muta et al. 2015), but we detected no specific difference in food intake during the dark cycle in the p54-S6K2 group (Fig. 3C).

In addition, p70-S6K1 mice showed greater 24-h activity than EGFP-control mice (Fig. 3D). No differences were observed in 24-h activity for p85-S6K1 and p54-S6K2
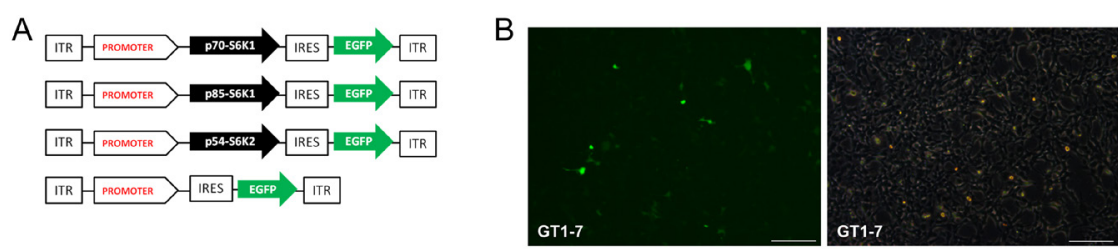

C
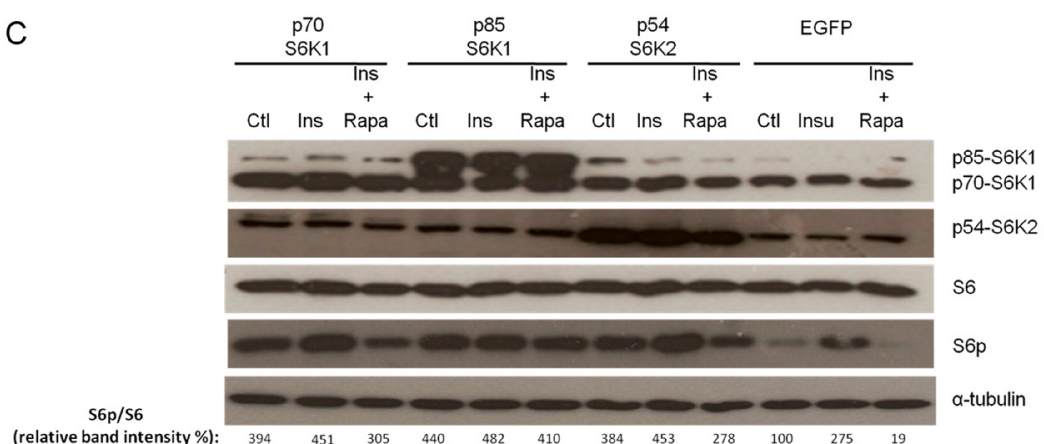

\section{Figure 1}

Validation of adenoviral vectors overexpressing different S6Ks isoforms in vitro. (A) Schematic representation of the targeting approach used for the construction of p70-S6K1, p85-S6K1, p54-S6K2, and EGFP adenovirus vectors. (B) GT1-7 hypothalamic cell lines transduced with Ad-p85-S6K1 using MOI: 10,000. Scale bars: $200 \mu \mathrm{m}$. (C) Western blot analysis of HEK293 cell lines infected with Ad-p70-S6K1, Ad-p85-S6K1, Ad-p54-S6K2 and Ad-EGFP. Cells were serumstarved for $2 \mathrm{~h}$ and then treated with rapamycin $(100 \mathrm{nM})$ for $15 \mathrm{~min}$ and insulin $(10 \mu \mathrm{g} / \mathrm{mL})$ for another $15 \mathrm{~min}$. S6p/S6 quantification is represented. Anti-S6K1, anti-S6K2, anti-S6, anti-pS6 and anti- $\alpha$-tubulin antibodies were used. A full colour version of this figure is available at https://doi.org/10.1530/JOE-19-0364. 

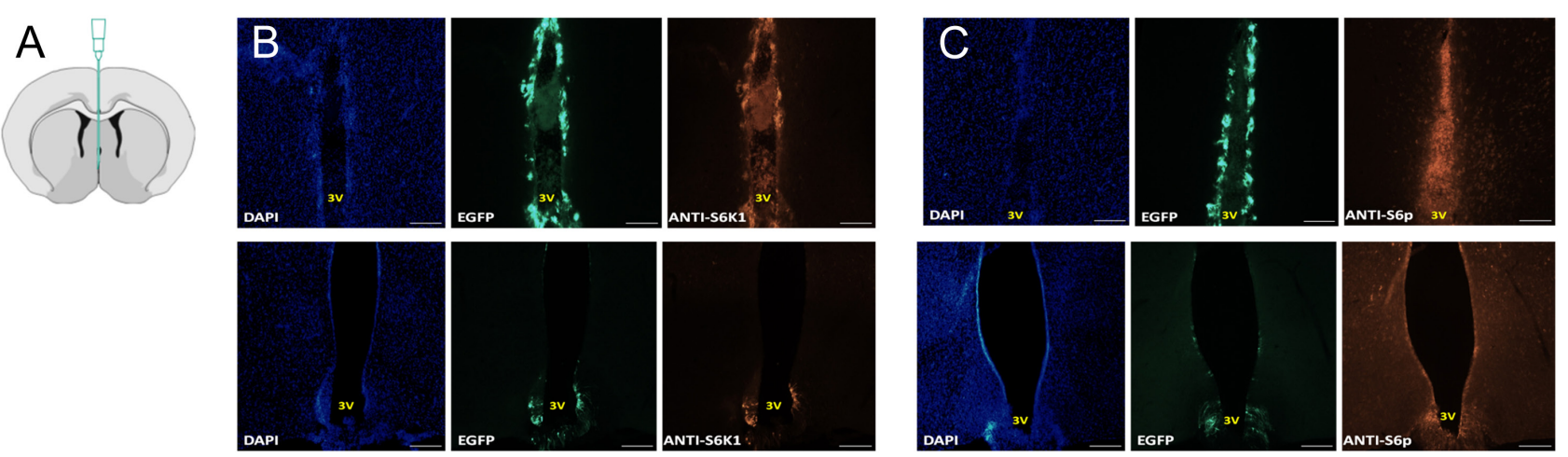

D
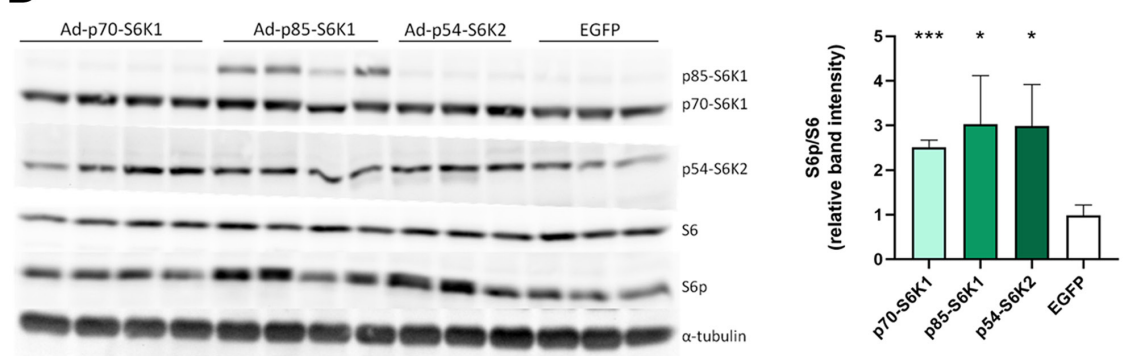

\section{Figure 2}

Overexpression of S6Ks isoforms in mice MBH. (A) Schematic diagram of the intracerebroventricular stereotaxic injection. (B) Immunofluorescence images illustrating nuclear labeling with DAPI (blue), EGFP labeling in green and S6K1 labeling in red on brain coronal sections ( $30 \mu \mathrm{m})$ of mice MBH injected with Ad-p85-S6K1 5 days after injection (top three panels) and 10 days after injection (bottom three panels). Scale bars: $200 \mu \mathrm{m}$.

(C) Immunofluorescence images illustrating nuclear labeling with DAPI (blue), EGFP (green) and phosphorylated-S6 (red) in brain coronal sections ( $30 \mu \mathrm{m}$ ) of mice MBH injected with Ad-p85-S6K1 5 days after injection (top three panels) and 10 days after injection (bottom three panels). Scale bars: $200 \mu \mathrm{m}$. (D) Western blot analysis of mice MBH injections with Ad-p70-S6K1, Ad-p85-S6K1, Ad-p54-S6K2 and Ad-EGFP. The graphic represents phosphorylated-S6 in hypothalamus. Anti-S6K1, anti-S6K2, anti-S6, anti-pS6 and anti- $\alpha$-tubulin antibodies were used. The bars represent the mean \pm s.E.M. Means significantly different as shown by unpaired $t$-tests $(* P<0.05)$ vs EGFP-control group.

mice (Fig. 3D). Also, we did not detect the differences in activity between S6Ks groups and EGFP-control in the dark cycle (Fig. 3E). No changes in the circadian pattern of respiratory exchange ratio (RER; Fig. 3F) or energy expenditure were observed (Fig. 3G).

To better understand the role of S6Ks controlling food consumption, we investigated whether mice would respond to overnight fasting $(12 \mathrm{~h})$ followed by a 2-h refeeding. S6Ks-injected mice had lower food consumption compared to the EGFP-control group after fasting (Fig. 3H), indicating that overexpression of S6Ks renders mice less sensitive to fasting.

Then, we evaluated the mRNA expression of important neuropeptides for feeding control and found that S6Ks-injected mice showed a decrease in the Agrp orexigenic gene (Fig. 3I) compared with EGFP-control. p70-S6K1- and p85-S6K1-injected mice also featured lower NPY levels (Fig. 3J). The POMC mRNA was equal to the EGFP-control mice, except for p85-S6K1-injected mice, which unexpectedly showed decreased expression compared with EGFP-control (Fig. 3K).

\section{Hypothalamic S6Ks modulate hepatic glucose metabolism}

As hypothalamic S6K1 may control glucose homeostasis (Ono et al. 2008, Smith et al. 2015), we sought peripheral changes resulting from the S6Ks modulation in the hypothalamus. Mice with overexpression of hypothalamic p70-S6K1 presented higher levels of fasting blood glucose compared with the EGFP-control group (Fig. 4A). However, no change in glucose tolerance assessed by ipGTT was observed comparing all groups (Fig. 4B and C).

To verify the potential changes in hepatic glucose production, we performed the intraperitoneal pyruvate tolerance test (ipPTT; Hughey et al. 2014). We found that only mice injected with p70-S6K1 presented increased blood glucose levels after the pyruvate challenge compared with EGFP-control (Fig. 4D and E), indicating a possible modulation of gluconeogenesis. Noteworthily, all groups showed higher levels of G6Pase mRNA compared with EGFP-control (Fig. 4F), indicating that hypothalamic S6Ks modulate peripheral glucose metabolism. 
A
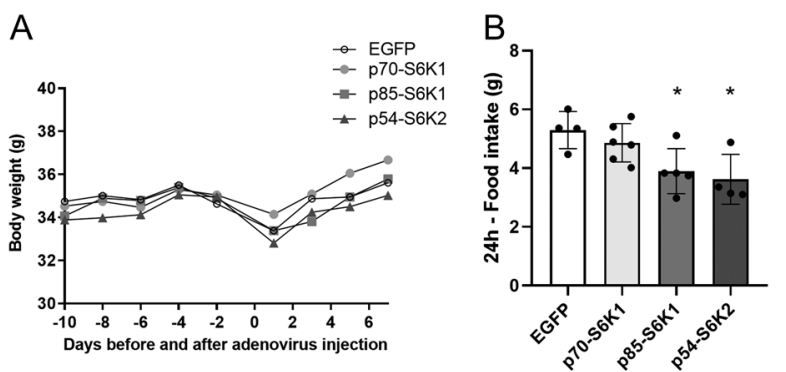

$\mathrm{E}$
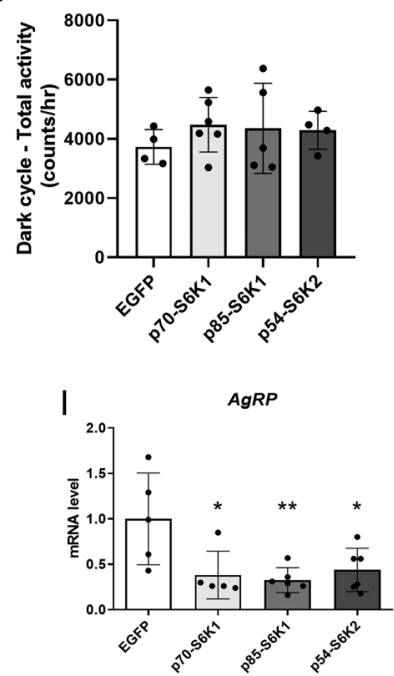

F
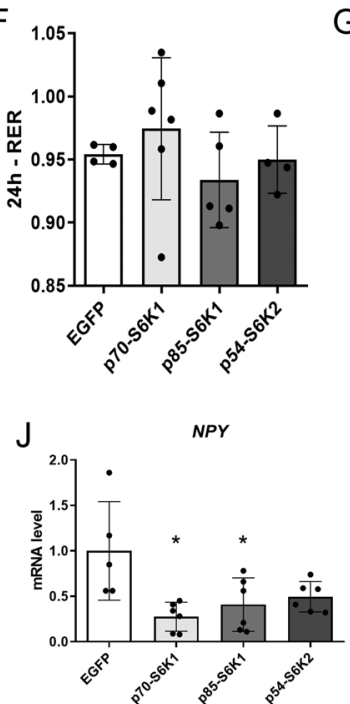
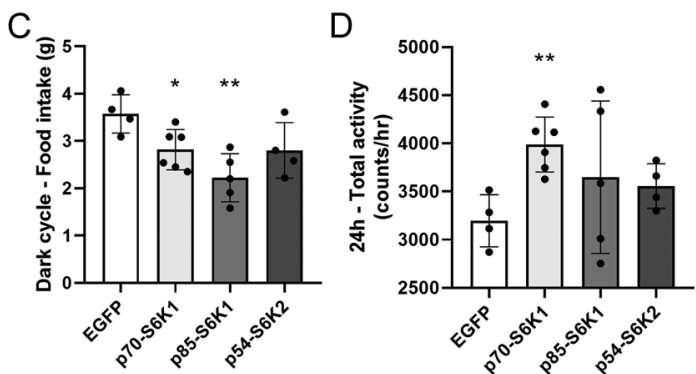

\section{G}
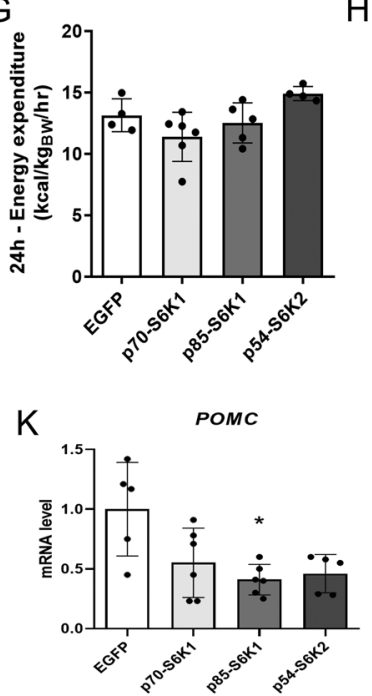

$\mathrm{H}$
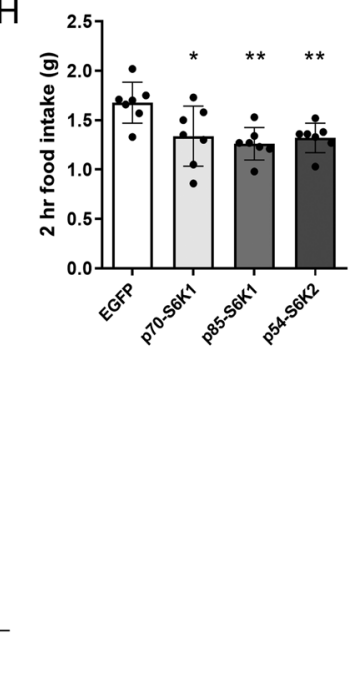

Figure 3

Hypothalamic S6Ks isoforms decrease food intake, fasting sensitivity and orexigenic neuropeptides levels. (A) Change in body weight (g) before and after MBH injections of Ad-EGFP (open circle), Ad-p70-S6K1 (filled circle), Ad-p85-S6K1 (filled square) and Ad-p54-S6K2 (filled triangle). (B) 24-h-food intake (g) and (C) dark cycle-food intake (g). (D) 24-h-total activity (counts/h) and (E) dark cycle-total activity (counts/h); (F) 24-h-RER (VCO ${ }_{2} / \mathrm{VO}_{2}$ ); (G) 24-h-energy expenditure $\left(\mathrm{kcal} / \mathrm{kg}_{\mathrm{BW}} / \mathrm{h}\right)$ of mice MBH injected with Ad-EGFP $(n=5)$, Ad-p70-S6K1 $(n=7)$, Ad-p85-S6K1 ( $\left.n=6\right)$ and Ad-p54-S6K2 $(n=6)$ measured 5 days after injections. (H) 2-h food intake (g) after 12-h-overnight fasting of mice MBH injected with Ad-EGFP $(n=7)$, Ad-p70-S6K1 ( $n=7)$, Ad-p85-S6K1 ( $n=7)$ and Ad-p54-S6K2 ( $n=7$ ) measured 12 days after injections. (I) AgRP-, (J) NPY-, (K) POMC mRNA levels of random-fed mice MBH injected with Ad-EGFP, Ad-p70-S6K1, Ad-p85-S6K1 and Ad-p54-S6K2 for 7 days. The bars represent the mean \pm S.E.M. Means significantly different as shown by unpaired $t$-tests $(* P<0.05 ; * \star P<0.01)$ vs EGFP-control group.

\section{S6K2 overexpression in AgRP neurons}

AgRP neurons are well-defined metabolic sensors in the ARH (Olofsson et al. 2013, Xu et al. 2018). To verify if S6K2 isoform plays a role in these specific neurons, we bilaterally injected Cre-dependent AAV-S6K2 into AgRP-Cre male and female mice (Fig. 5A).

We initially tested Cre-dependent expression of pAAV-S6K2 and pAAV-EGFP plasmids by co-transfecting HEK293 cells with a Cre-expressing plasmid (pCAG; Fig. 5B). Since S6K2 and EGFP genes were fused with FLAG peptide DNA sequence, the expression of S6K2 $(60 \mathrm{kDa})$ and EGFP $(32.7 \mathrm{kDa})$ in cells expressing Cre was verified using an anti-FLAG antibody (Fig. 5B). The expression of FLAG-S6K2 in pAAV-S6K2 Cre-cells was also detected above the endogenous $\mathrm{S} 6 \mathrm{~K} 2$ protein (Fig. 5B). In vivo experiments were performed in two different cohorts of male mice and two different cohorts of female mice. We performed 12 bilateral injections of the AAV-S6K2 vector into AgRP-Cre males and 14 bilateral injections into AgRP-Cre females. We obtained six males and eight females with unilateral or bilateral injections into AgRP neurons (Fig. 5C and D), as confirmed by FLAG expression by immunohistochemical analysis (Fig. 5E and F). Since injections were variable and thus resulting in a small number of mice obtained from each cohort, data were analyzed combining both cohorts.

In male mice, we found no differences in body weight between control and AgRP-S6K2 groups before and after bilateral injections (Fig. 6A). There was no difference in food intake measured for three consecutive days (Fig. 6B), neither when the dark cycle was separately analyzed (Fig. 6C). No changes in total 
A

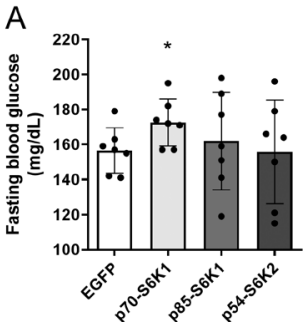

B
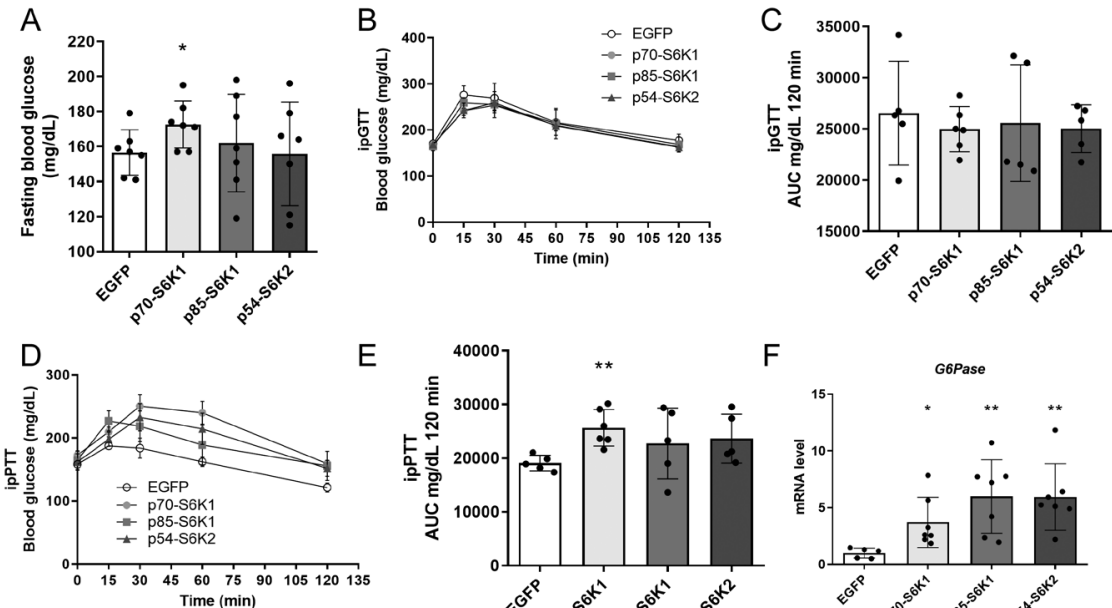

E

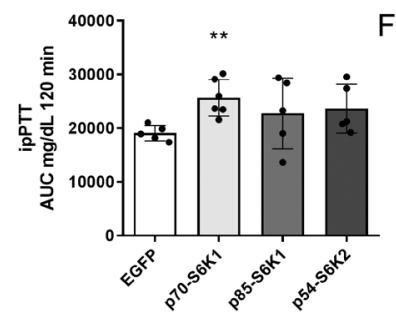

\section{Figure 4}

Hypothalamic p70-S6K1 isoform alters hepatic glucose production. (A) Blood glucose of mice MBH injected with Ad-EGFP $(n=7)$, Ad-p70-S6K1 $(n=7)$, Ad-p85-S6K1 $(n=7)$ and Ad-p54-S6K2 $(n=7)$ for 8 days, measured after 12-h overnight fasting. (B) Blood glucose curve and (C) area under the curve graph of intraperitoneal glucose tolerance test (ipGTT) performed in mice MBH injected with Ad-EGFP, Ad-p70-S6K1, Ad-p85-S6K1, and Ad-p54-S6K2. (D) Blood glucose curve and (E) area under the curve graph of intraperitoneal pyruvate tolerance test (ipPTT) performed in mice $\mathrm{MBH}$ injected with Ad-EGFP, Ad-p70-S6K1, Ad-p85-S6K1, and Ad-p54-S6K2, 8 days after injections. (F) G6Pase mRNA levels of random fed mice MBH injected with Ad-EGFP, Ad-p70-S6K1, Ad-p85-S6K1, and Ad-p54-S6K2 for 14 days. The bars represent the mean \pm s.E.M. Means significantly different as shown by unpaired $t$-tests ( $* P<0.05$; $* * P<0.01$ ) vs EGFP-control group.

activity (Fig. 6D), RER (Fig. 6E), or energy expenditure (Fig. 6F) were found. When we separately analyzed data, there were no changes in RER and energy expenditure in the dark cycle (data not shown). No effect was observed in the 2 -h refeeding test (overnight fasting, Fig. 6G). However, when male cohorts were evaluated separately, we observed that AgRP-S6K2 male mice in the second cohort showed increased food intake when compared with control mice (AgRP-S6K2, $1.348 \pm 0.1621$ vs control, $0.6275 \pm 0.1585 \mathrm{~g}, n=4$, $P=0.0192$ ), suggesting variability in injection amount.

In female mice, body weight was equal between AgRP-S6K2 and control groups on the day we started CLAMS measurements (Fig. 7A). Likewise, no differences in food intake measured for three consecutive days (Fig. 7B) or in the dark cycle (Fig. 7C) were found. There was no change in total activity (Fig. 7D and E), RER (Fig. 7F), nor in energy expenditure (Fig. 7G), even in the dark cycle, when data were separately analyzed (data not shown). However, females AgRP-S6K2 presented increased food intake in the 2 -h refeeding test (Fig. $7 \mathrm{H}$ ) and curiously, lower body weight (AgRP-S6K2, 20.54 \pm 0.5067 g, $n=8$ vs control, $22.71 \pm 0.8134 \mathrm{~g}, n=15, P=0.0345)$ after CLAMS compared with control. However, analysis of delta body weight gain before and after CLAMS indicates no significant differences (Fig. 7I). Due to the lower body weight presented by the AgRP-S6K2 female mice, we also analyzed refeeding data corrected by body weight and

A

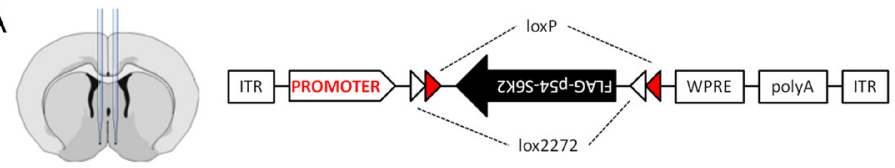

B
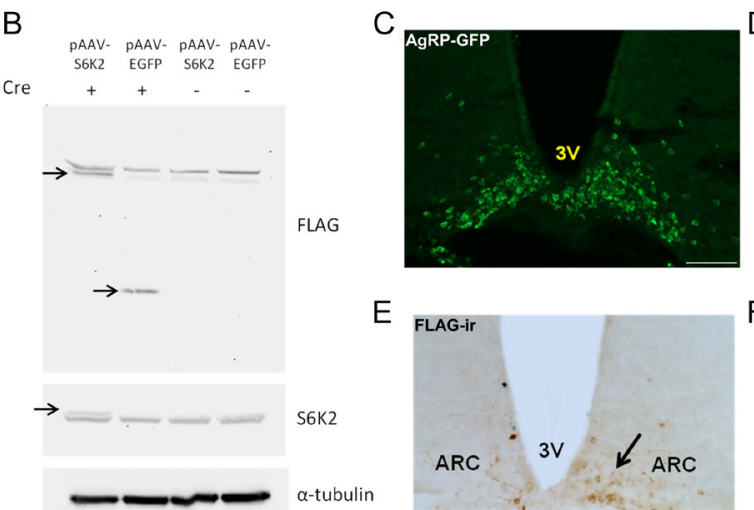

E

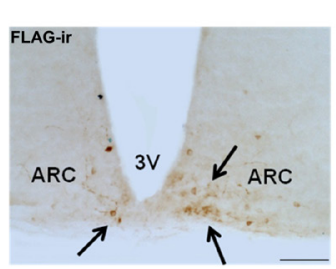

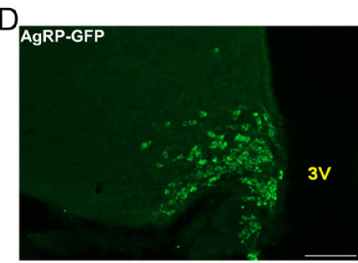

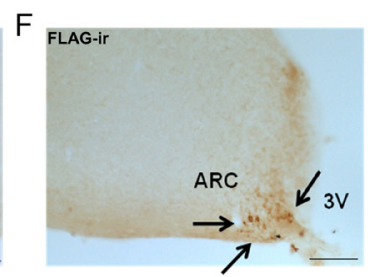

Figure 5

Validation of Cre-dependent AVV8-S6K2 in vitro and in vivo. (A) Schematic diagram of the bilateral stereotaxic surgery in ARH and targeting approach for p54-S6K2 Cre-dependent AAV vectors. (B) Western blot analysis of HEK293 cells co-transfected with Cre-expressing plasmid (pCAG) or non-Cre-expressing plasmid (pShires) and pAAV-S6K2 or PAAV-EGFP. Anti-FLAG, anti-S6K2, and anti- $\alpha$-tubulin antibodies were used. (C and D) AgRP-GFP neurons (green) in ARH hypothalamus and tuberal region of AgRP-Crepositive mice. Scale bars: $100 \mu \mathrm{m}$. (E and F) Immunohistochemical analysis showing the expression of FLAG (p54-S6K2) in AgRP neurons in the ARH of AgRP-Cre mice. Scale bars: $100 \mu \mathrm{m}$. A full colour version of this figure is available at https://doi.org/10.1530/JOE-19-0364. 


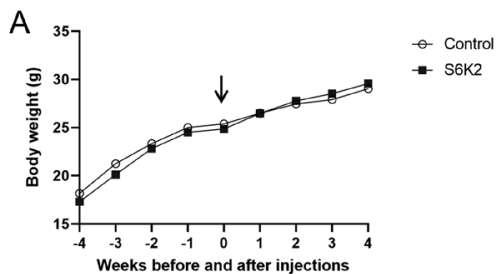

B
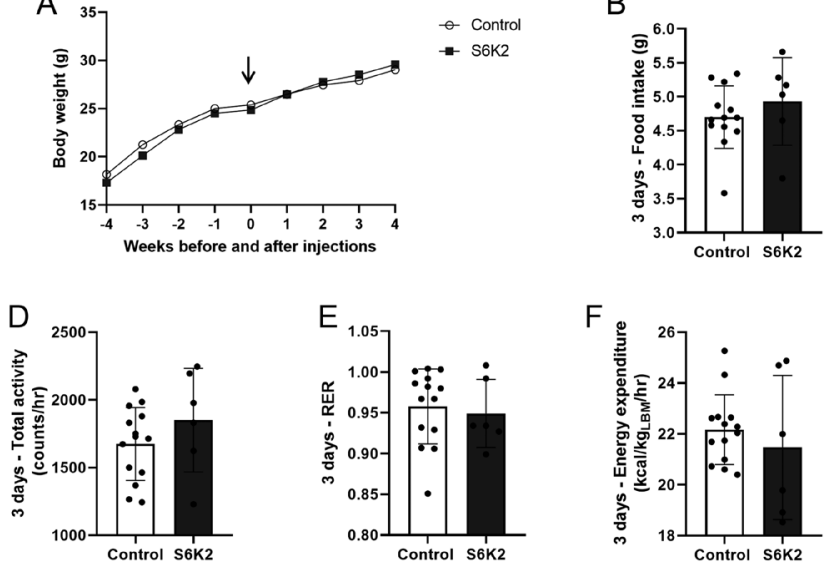

we noticed that AgRP-S6K2 female mice were still more sensitive to fasting (S6K2, $0.05597 \pm 0.006730 \mathrm{~g} / \mathrm{gBW}, n=8$ vs control, $0.03336 \pm 0.005576 \mathrm{~g} / \mathrm{gBW}, n=15, P=0.0214$ ). Altogether, these data indicate that S6K2 plays a role in the control of fasting sensitivity in AgRP neurons.

\section{Discussion}

The mTORC1/S6Ks role in metabolism control is divergent, especially regarding central and peripheral functions. In obesity, this signaling pathway is overactivated in peripheral tissues (Um et al. 2004, Khamzina et al. 2005), whereas in the hypothalamus, mTORC1/S6K activation is necessary for leptin- and insulin-mediated anorexigenic effects (Cota et al. 2006, 2008, Blouet et al. 2008, Muta et al. 2015). Our data are in agreement with these studies since decreased food intake, observed in mice with S6Ks overexpression in periventricular and ARH regions, was followed by decreased AgRP and NPY mRNA levels. Noteworthily, we also found that p54-S6K2 isoform presents anorexigenic effects in hypothalamic neurons. Unexpectedly, we noticed a decreased expression of POMC mRNA in p85S6K1 mice. Since POMC processing can be affected by insulin and leptin signaling, dietary factors and energy balance, this finding may not reflect the levels of the biologically active neuropeptides after POMC processing (Wardlaw 2011).

Our data indicate that mice with hypothalamic overexpression of S6Ks presented lower sensitivity to fasting. This effect can be mediated by the action of hormones, like leptin and insulin, since S6Ks signaling is necessary for the anorexigenic effects of these hormones in the hypothalamus (Blouet et al. 2008,
C

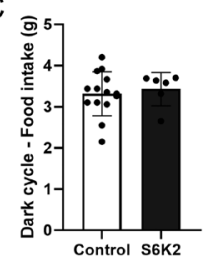

G

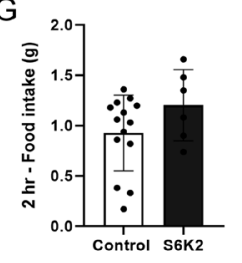

\section{Figure 6}

Effects of S6K2 overexpression in AgRP neurons in male mice. (A) Change in body weight (g) in male mice before and after bilateral injections of AAV-empty-control and AAV-S6K2. Arrow represents the day of injections. (B) 3 days- and (C) dark cycle-food intake; (D) 3 days-total activity (counts/h); (E) 3 days-RER $\left(\mathrm{VCO}_{2} / \mathrm{VO}_{2}\right) ;(\mathrm{F}) 3$ days-energy expenditure $\left(\mathrm{kcal} / \mathrm{kg}_{\text {LвM }} / \mathrm{h}\right) ;(\mathrm{G})$ 2-h-food intake (g) after $16 \mathrm{~h}$ of fasting in male mice. Data were analyzed combining cohorts 1 and 2. Control $n=14$; AgRP-S6K2 $n=6$. The bars represent the mean \pm S.E.M. Means significantly different as shown by unpaired $t$-tests $(* P<0.05)$ vs control group.

Cota et al. 2008, Muta et al. 2015). It is possible that the increase in hypothalamic S6Ks may improve leptin and insulin signaling after a fasting period, leading to lower food intake.

The change in feeding behavior observed in S6Ks groups was not followed by changes in body weight. The overexpression of proteins mediated by adenoviral vectors consists essentially of an acute approach; thus, changes in food intake may not reflect changes in body weight at this point. It has been demonstrated that rapamycin treatment takes at least 2 weeks to alter body weight in mice (Yang et al. 2012). Furthermore, we noticed no differences in energy expenditure in S6Ks groups, despite p70-S6K1 group demonstrating higher locomotor activity. However, a study demonstrated that the effect of activity on daily energy expenditure can be minimal in mice (O'Neal et al. 2017).

The adenovirus-mediated S6Ks overexpression in periventricular and ARH regions of the hypothalamus may be leading to important systemic changes, mainly involving glucose metabolism. One of the important insulin actions in the CNS is the suppression of hepatic glucose production. This effect is blocked by a highfat diet (HFD) consumption, due to the development of insulin resistance (Ono et al. 2008). Our findings indicate that hypothalamic p70-S6K1 overexpression increased blood glucose levels after fasting and pyruvate challenges, followed by increased hepatic G6Pase mRNA levels, supporting an increase in gluconeogenesis and impairment of hepatic insulin signaling. Increased levels of G6Pase mRNA in the liver of mice with p70-S6K1, p85-S6K1, and p54-S6K2 overexpression give additional support for the role of hypothalamic S6Ks isoforms in controlling peripheral glucose metabolism. Our data are in agreement with a previous study, whose authors 
A
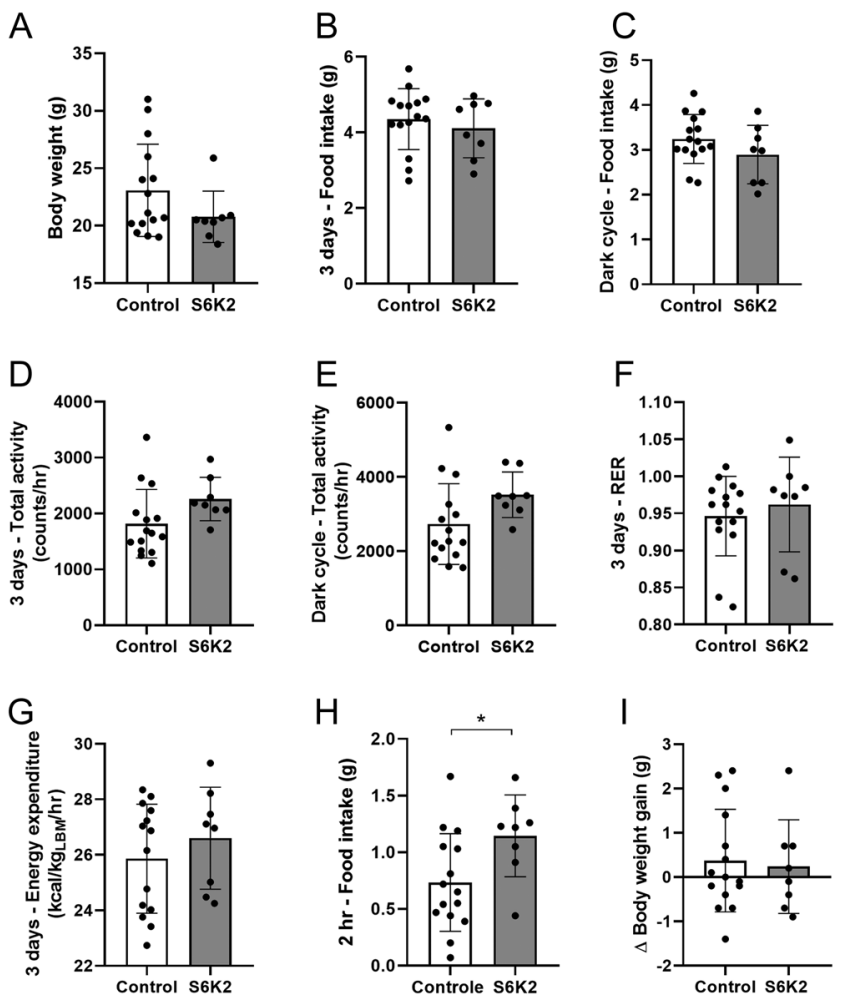

\section{Figure 7}

Effects of S6K2 overexpression in AgRP neurons in female mice. (A) Body weight $(\mathrm{g})$ in female mice after bilateral injections of AAV-empty-control and AAV-S6K2 before CLAMS analysis. (B) 3 days- and (C) dark cycle-food intake; (D) 3 days- and (E) dark cycle-total activity (counts/h); (F) 3 days-RER $\left(\mathrm{VCO}_{2} / \mathrm{VO}_{2}\right) ;(\mathrm{G}) 3$ days-energy expenditure $\left(\mathrm{kcal} / \mathrm{kg}_{\mathrm{LBM}} / \mathrm{h}\right) ;(\mathrm{H}) 2-\mathrm{h}$ food intake ( $\mathrm{g}$ ) after $16 \mathrm{~h}$ of fasting in female mice. (I) Delta $(\Delta)$ body weight gain $(\mathrm{g})$ in female mice after CLAMS analysis. Data were analyzed combining cohorts 1 and 2. Control $n=15$; S6K $2 n=8$. The bars represent the mean \pm S.E.M. Means significantly different as shown by unpaired $t$-tests $\left({ }^{*} P<0.05\right)$ vs control group.

showed that hypothalamic p70-S6K1 overactivation impairs hypothalamic insulin to suppress hepatic glucose production, a similar effect observed in rats fed with an HFD (Ono et al. 2008). Moreover, an improvement in systemic glucose metabolism is noticed when DEPTOR, a negative mTORC1 regulator, is overexpressed in the MBH (Caron et al. 2016).

Studies involving mTORC1/S6Ks and metabolic control are challenge since they are strictly related to the nutritional and hormonal status of each animal and a fine modulation of this pathway is reported (Hu et al. 2016). Although leptin and insulin require hypothalamic mTORC1/S6Ks activation for their anorexigenic functions, the overactivation of this pathway, as occurs in obesity, is harmful. This has been explained due to a negative feedback between S6K1 and IRS-1, in which S6K1, when overactivated, is able to phosphorylate IRS-1 serine residues (Tremblay et al. 2007), leading to IRS-1 inactivation and degradation and to the development of insulin resistance (Um et al. 2004, Zhang et al. 2008). Therefore, in obesity, impairment of hypothalamic mTORC1 signaling occurs together with the development of hyperphagia, body weight gain and leptin and insulin resistance (Cota et al. 2008). This negative feedback loop also explains the impairment of insulin signaling in the hypothalamus, which leads to increased hepatic glucose production and hepatic insulin resistance (Ono et al. 2008), as we reported in mice with increased expression of S6Ks, especially of p70-S6K1, in periventricular and ARH.

The adenoviral vectors approach used in this study has limitations because it causes a robust increase in gene expression in a short period of time in unidentified cells (Smith et al. 2015). The overexpression of target genes can increase the baseline activity of the coding protein, leading to a non-physiological response. The hypothalamus is comprised of heterogeneous populations of neurons expressing neuropeptides with opposite functions, for example, AgRP/NPY and POMC. The nonselective transduction of the S6Ks impacts the analysis of specific contributions of each cell type since hypothalamic mTORC1/S6Ks activation is neuron type and context dependent (Hu et al. 2016).

In order to surpass these limitations, we used a Cre-loxP system in a conditional approach to investigate a possible role of $\mathrm{S} 6 \mathrm{~K} 2$ isoform selectively in AgRP neurons, due to the lack of data on this specific isoform. AgRP neurons are leptin and insulin targets to control energy balance and glucose homeostasis (Könner et al. 2007, Xu et al. 2018). Most of AgRP neurons are localized outside the blood-brain barrier and are highly expressed in the medial or periventricular ARH, turning them important neurons that quickly respond to hormonal and nutritional statuses (Olofsson et al. 2013, Xu et al. 2018). Furthermore, phosphorylated mTOR and S6K1 are found in nearly 90\% of ARH NPY/AgRP neurons and only in $45 \%$ of POMC/CART neurons (Cota et al. 2006).

Our findings support that S6K2 exerts an important effect, increasing fasting sensitivity when overexpressed in AgRP neurons. This may seem counterintuitive since we found that hypothalamic p54-S6K2-unspecific overexpression decreased the sensitivity to fasting. However, it is believed that the role of mTOR/S6Ks signaling in the hypothalamus depends on the energy status and varies according to the type of neuron reached (Hu et al. 2016). A study showed increased 
phosphorylation of S6 in AgRP neurons upon fasting, while mTOR/S6Ks signaling was decreased in other hypothalamic nuclei, as ventromedial (Villanueva et al. 2009). All these findings together support that the role of mTOR/S6Ks signaling in hypothalamus depends on the neurons in which it is activated.

It is worth mentioning that using a Cre-dependent approach and stereotaxic injections to specifically target AgRP neurons resulted in an inconsistent and low number of cells overexpressing S6K2. This may also explain the variability of the data between the groups and possibly the lack of changes in most of the evaluated metabolic parameters. However, authors of previous studies have shown that the deletion of S6K1 in AgRP and POMC neurons did not alter body weight or feeding behavior in mice (Smith et al. 2015). On the other hand, S6K1 signaling in AgRP neurons controls muscle insulin sensitivity and glucose levels, highlighting its role in peripheral metabolism control (Smith et al. 2015). Further studies will be necessary to assess the role of S6K2 isoform in the entire AgRP population using mouse lines.

Here we demonstrated a role for S6K2 signaling in AgRP neurons controlling feeding behavior, in agreement with the facts that mTOR/S6Ks signaling, in specific neurons, controls key peripheral functions and that insulin signaling in AgRP neurons is necessary to control hepatic glucose production (Könner et al. 2007). We highlight the need for further investigation into the S6Ks role, especially S6K2, regarding metabolism control and their central and systemic roles.

\section{Conclusions}

We showed new insights into the function of different S6Ks isoforms, especially p54-S6K2, in the hypothalamic control of energy homeostasis. Our findings also indicate that an increase in hypothalamic mTORC1/S6Ks signaling, especially via p70-S6K1 isoform, impairs systemic glucose metabolism. The present study contributes with new evidence on the S6Ks role in hypothalamic neurons for controlling metabolism.

\section{Declaration of interest}

The authors declare that there is no conflict of interest that could be perceived as prejudicing the impartiality of the research reported.

\section{Funding}

This research has been funded by The São Paulo Research Foundation, FAPESP (grant numbers: F M S, 2012/13558-7 and 2018/14818-9; R M N B, 2016/06457-0; fellowship numbers: M R T, 2013/22696-7, 2015/24475-3 and 2018/07018-6; I C B P 2015/003111) and from The Brazilian National Council for Scientific and Technological Development, CNPq (F M S, 447553/2014-3). C F E is funded by NIH grants (HD069702 and HD092855) and the University of Michigan Animal Phenotyping Core is funded by the following NIH-P30 grants: DK020572 (MDRC), DK089503 (MNORC), and 1U2CDK110678-01 (Mi-MMPC).

\section{Author contribution statement}

M R T has written the manuscript and headed all experiments execution. M R T, S F L, T F, and I C B P have performed experiments and revised the manuscript. F M S, P O P, M A T, and R M N B have designed cell culture and adenovirus experiments and revised the manuscript. F M S, C F E, and C S M have designed A A V experiments and revised the manuscript.

\section{Acknowledgments}

The authors would like to thank Susan Allen and Emily Henson for animal care and histology and the many colleagues they have worked with over the years. They thank professor Carlos Meck from the University of São Paulo for kindly giving them the pShires plasmid.

\section{References}

Armelini MG, Muotri AR, Marchetto MCN, De Lima-Bessa KM, Sarasin A \& Menck CFM 2005 Restoring DNA repair capacity of cells from three distinct diseases by XPD gene-recombinant adenovirus. Cancer Gene Therapy 12 389-396. (https://doi. org/10.1038/sj.cgt.7700797)

Blouet C, Ono H \& Schwartz GJ 2008 Mediobasal hypothalamic P70 S6 kinase 1 modulates the control of energy homeostasis. Cell Metabolism 8 459-467. (https://doi.org/10.1016/j.cmet.2008.10.004)

Bouret SG \& Simerly RB 2004 Minireview: leptin and development of hypothalamic feeding circuits. Endocrinology 145 2621-2626. (https:// doi.org/10.1210/en.2004-0231)

Caron A, Labbé SM, Lanfray D, Blanchard PG, Villot R, Roy C, Sabatini DM, Richard D \& Laplante M 2016 Mediobasal hypothalamic overexpression of DEPTOR protects against high-fat diet-induced obesity. Molecular Metabolism 5 102-112. (https://doi.org/10.1016/j. molmet.2015.11.005)

Cota D, Matter EK, Woods SC \& Seeley RJ 2008 The role of hypothalamic mammalian target of rapamycin complex 1 signaling in dietinduced obesity. Journal of Neuroscience 28 7202-7208. (https://doi. org/10.1523/JNEUROSCI.1389-08.2008)

Cota D, Proulx K, Blake Smith KA, Kozma SC, Thomas G, Woods SC \& Seeley RJ 2006 Hypothalamic mTOR signaling regulates food intake. Science 312 927-930. (https://doi.org/10.1126/science.1124147)

Dodd GT \& Tiganis T 2017 Insulin action in the brain: roles in energy and glucose homeostasis. Journal of Neuroendocrinology 29 e12513. (https://doi.org/10.1111/jne.12513)

$\mathrm{Hu}$ F, Xu Y \& Liu F 2016 Hypothalamic roles of mTOR complex I: integration of nutrient and hormone signals to regulate energy homeostasis. American Journal of Physiology. Endocrinology and Metabolism 310 E994-E1002. (https://doi.org/10.1152/ ajpendo.00121.2016) https://joe.bioscientifica.com

https://doi.org/10.1530/JOE-19-0364 (c) 2020 Society for Endocrinology Published by Bioscientifica Ltd. Printed in Great Britain 
Hughey CC, Wasserman DH, Lee-Young RS \& Lantier L 2014 Approach to assessing determinants of glucose homeostasis in the conscious mouse. Mammalian Genome 25 522-538. (https://doi.org/10.1007/ s00335-014-9533-z)

Ignacio-Souza LM, Bombassaro B, Pascoal LB, Portovedo MA, Razolli DS, Coope A, Victorio SC, De Moura RF, Nascimento LF, Arruda AP, et al. 2014 Defective regulation of the ubiquitin/proteasome system in the hypothalamus of obese male mice. Endocrinology 155 2831-2844. (https://doi.org/10.1210/en.2014-1090)

James WPT, Rigby N \& Leach R 2006 Obesity and the metabolic syndrome: the stress on society. Annals of the New York Academy of Sciences 1083 1-10. (https://doi.org/10.1196/annals.1367.002)

Kahn BB \& Myers MG 2006 mTOR tells the brain that the body is hungry. Nature Medicine 12 615-617. (https://doi.org/10.1038/ nm0606-615)

Khamzina L, Veilleux A, Bergeron S \& Marette A 2005 Increased activation of the mammalian target of rapamycin pathway in liver and skeletal muscle of obese rats: possible involvement in obesitylinked insulin resistance. Endocrinology 146 1473-1481. (https://doi. org/10.1210/en.2004-0921)

Könner AC, Janoschek R, Plum L, Jordan SD, Rother E, Ma X, Xu C, Enriori P, Hampel B, Barsh GS, et al. 2007 Insulin action in AgRPexpressing neurons is required for suppression of hepatic glucose production. Cell Metabolism 5 438-449. (https://doi.org/10.1016/j. cmet.2007.05.004)

Krashes MJ, Lowell BB \& Garfield AS 2016 Melanocortin-4 receptorregulated energy homeostasis. Nature Neuroscience 19 206-219. (https://doi.org/10.1038/nn.4202)

Muta K, Morgan DA \& Rahmouni K 2015 The role of hypothalamic mTORC1 signaling in insulin regulation of food intake, body weight, and sympathetic nerve activity in male mice. Endocrinology 156 1398-1407. (https://doi.org/10.1210/en.2014-1660)

Myers MG \& Olson DP 2012 Central nervous system control of metabolism. Nature 491 357-363. (https://doi.org/10.1038/nature11705)

Nardella C, Lunardi A, Fedele G, Clohessy JG, Alimonti A, Kozma SC, Thomas G, Loda M \& Pandolfi PP 2011 Differential expression of S6K2 dictates tissue-specific requirement for S6K1 in mediating aberrant mTORC1 signaling and tumorigenesis. Cancer Research $\mathbf{7 1}$ 3669-3675. (https://doi.org/10.1158/0008-5472.CAN-10-3962)

O’Neal TJ, Friend DM, Guo J, Hall KD \& Kravitz AV 2017 Increases in physical activity result in diminishing increments in daily energy expenditure in mice. Current Biology 27 423-430. (https://doi. org/10.1016/j.cub.2016.12.009)

Olofsson LE, Unger EK, Cheung CC \& Xu AW 2013 Modulation of AgRPneuronal function by SOCS3 as an initiating event in diet-induced hypothalamic leptin resistance. PNAS 110 E697-E706. (https://doi. org/10.1073/pnas.1218284110)

Ono H, Pocai A, Wang Y, Sakoda H, Asano T, Backer JM, Schwartz GJ \& Rossetti L 2008 Activation of hypothalamic S6 kinase mediates diet-induced hepatic insulin resistance in rats. Journal of Clinical Investigation 118 2959-2968. (https://doi.org/10.1172/JCI34277)

Pardo OE \& Seckl MJ 2013 S6K2: the neglected S6 kinase family member. Frontiers in Oncology 3 191. (https://doi.org/10.3389/fonc.2013.00191)

Pavan ICB, Yokoo S, Granato DC, Meneguello L, Carnielli CM, Tavares MR, do Amaral CL, de Freitas LB, Paes Leme AF, Luchessi AD, et al. 2016 Different interactomes for p70-S6K1 and p54-S6K2 revealed by proteomic analysis. Proteomics 16 2650-2666. (https://doi. org/10.1002/pmic.201500249)
Pende M, Um SH, Mieulet V, Sticker M, Goss VL, Mestan J, Mueller M, Fumagalli S, Kozma SC \& Thomas G 2004 S6K1-/-/S6K2-/- mice exhibit perinatal lethality and rapamycin-sensitive 5 '-terminal oligopyrimidine mRNA translation and reveal a mitogen-activated protein kinase-dependent S6 kinase pathway. Molecular and Cellular Biology 24 3112-3124. (https://doi.org/10.1128/mcb.24.8.31123124.2004)

Schneeberger M, Gomis R \& Claret M 2014 Hypothalamic and brainstem neuronal circuits controlling homeostatic energy balance. Journal of Endocrinology 220 T25-T46. (https://doi.org/10.1530/JOE13-0398)

Smith MA, Katsouri L, Irvine EE, Hankir MK, Pedroni SMA, Voshol PJ, Gordon MW, Choudhury AI, Woods A, Vidal-Puig A, et al. 2015 Ribosomal S6K1 in POMC and AgRP neurons regulates glucose homeostasis but not feeding behavior in mice. Cell Reports 11 335-343. (https://doi.org/10.1016/j.celrep.2015.03.029)

Tavares MR, Pavan ICB, Amaral CL, Meneguello L, Luchessi AD \& Simabuco FM 2015 The S6K protein family in health and disease. Life Sciences 131 1-10. (https://doi.org/10.1016/j.lfs.2015.03.001)

Timper K \& Brüning JC 2017 Hypothalamic circuits regulating appetite and energy homeostasis: pathways to obesity. Disease Models \& Mechanisms 10 679-689. (https://doi.org/10.1242/dmm.026609)

Tremblay A \& Lachance É 2017 Tackling obesity at the community level by integrating healthy diet, movement and non-movement behaviours. Obesity Reviews 18 (Supplement 1) 82-87. (https://doi. org/10.1111/obr.12504)

Tremblay F, Brule S, Hee Um S, Li Y, Masuda K, Roden M, Sun XJ, Krebs M, Polakiewicz RD, Thomas G, et al. 2007 Identification of IRS-1 Ser-1101 as a target of S6K1 in nutrient- and obesity-induced insulin resistance. PNAS 104 14056-14061. (https://doi.org/10.1073/ pnas.0706517104)

Um SH, Frigerio F, Watanabe M, Picard F, Joaquin M, Sticker M, Fumagalli S, Allegrini PR, Kozma SC, Auwerx J, et al. 2004 Absence of S6K1 protects against age- and diet-induced obesity while enhancing insulin sensitivity. Nature 431 200-205. (https://doi.org/10.1038/ nature02866)

Villanueva EC, Münzberg H, Cota D, Leshan RL, Kopp K, IshidaTakahashi R, Jones JC, Fingar DC, Seeley RJ \& Myers MG 2009 Complex regulation of mammalian target of rapamycin complex 1 in the basomedial hypothalamus by leptin and nutritional status. Endocrinology 150 4541-4551. (https://doi.org/10.1210/en.20090642)

Wardlaw SL 2011 Hypothalamic proopiomelanocortin processing and the regulation of energy balance. European Journal of Pharmacology 660 213-219. (https://doi.org/10.1016/j. ejphar.2010.10.107)

Xu J, Bartolome CL, Low CS, Yi X, Chien CH, Wang P \& Kong D 2018 Genetic identification of leptin neural circuits in energy and glucose homeostases. Nature 556 505-509. (https://doi.org/10.1038/s41586018-0049-7)

Yang SB, Tien AC, Boddupalli G, Xu AW, Jan YN \& Jan LY 2012 Rapamycin ameliorates age-dependent obesity associated with increased mTOR signaling in hypothalamic POMC neurons. Neuron 75 425-436. (https://doi.org/10.1016/j.neuron.2012.03.043)

Zhang J, Gao Z, Yin J, Quon MJ \& Ye J 2008 S6K directly phosphorylates IRS-1 on Ser-270 to promote insulin resistance in response to TNF- $\alpha$ signaling through IKK2. Journal of Biological Chemistry 28335375 35382. (https://doi.org/10.1074/jbc.M806480200) https://joe.bioscientifica.com https://doi.org/10.1530/JOE-19-0364
(C) 2020 Society for Endocrinology Published by Bioscientifica Ltd. Printed in Great Britain
Received in final form 23 September 2019

Accepted 26 September 2019

Accepted Manuscript published online 26 September 2019 